\title{
Nasal Nitric Oxide May Not Differentiate Primary Ciliary Dyskinesia from Certain Primary Immunodeficiencies
}

\author{
Jessica Saunders ${ }^{1}$, Michael O'Connor ${ }^{2}$, and Evans Machogu ${ }^{1}$ \\ ${ }^{1}$ Indiana University School of Medicine \\ ${ }^{2}$ Vanderbilt University Medical Center
}

February 24, 2022

\begin{abstract}
The diagnosis of primary ciliary dyskinesia (PCD) is made through a combination of clinical features supported by a panel of diagnostic tests. Our cases highlight the similarities in the clinical presentation of patients with the specific immunodeficiency activated phosphatidylinositol 3-kinase delta syndrome 1 (APDS1 or PIK3CD) and PCD. We highlight the importance of repeating nasal nitric oxide testing ( $\mathrm{nNO}$ ) when PCD has not been confirmed by genetic or ciliary electron micrograph (EM) analysis in the setting of an expanded suppurative lung disease differential that includes considerations for immunodeficiency as well as PCD.
\end{abstract}

\section{Introduction}

Primary ciliary dyskinesia (PCD) is a ciliopathy characterized by chronic and recurrent sinopulmonary infections due to impaired function of the motile cilia[1]. The diagnosis of PCD requires a compatible clinical phenotype accompanied by supportive diagnostic studies. A low nasal nitric oxide (nNO), $<77 \mathrm{~nL} / \mathrm{min}$, in a patient with compatible clinical features, has a high sensitivity $(>98 \%)$ and specificity $(>99.9 \%)$ for establishing a diagnosis of $\mathrm{PCD}[1]$. However, low nNO levels have been reported in other disease processes such as cystic fibrosis and primary immunodeficiencies (PID) [2] and can therefore not solely be used to make a conclusive diagnosis of PCD. We report two patients with low nNO values and overlapping clinical symptoms of PCD who were later determined to have the specific immunodeficiency activated phosphatidylinositol 3kinase delta syndrome 1 (APDS1) or PIK3CD.

\section{Case 1}

An 8-year-old male born at term had a history of unexplained neonatal respiratory distress with a brief oxygen requirement. He had multiple hospitalizations for recurrent pneumonia, recurrent otitis media, sinusitis and wheezing. His evaluation included a normal chromosomal microarray and sweat chloride test with an immunologic work up revealing a nonspecific polyclonal gammopathy managed with prophylactic antibiotics.

Computed tomography (CT) scan of his sinuses and chest at the age of 2 years revealed pansinusitis as well as multiple areas of mucus plugging, atelectasis and tree in bud opacities. Repeat imaging at the age of 7 years showed progressive lung disease and development of bronchiectasis. Bronchoscopy on two separate occasions demonstrated mucus plugging and nodular mucosa with Moraxella catarrhalis and Hemophilus influenza bacteria isolated from cultures. Measurements of $\mathrm{nNO}$ were obtained at various points as part of an evaluation for PCD (Table ). PCD genetic testing returned variants of unknown significance (VUS) in both DNAH8 and DRC1 genes. 
His symptoms of cough and rhinorrhea waxed and waned and were uncharacteristic of PCD. Additionally, he had recurrent abdominal pain, and abnormal stools. With the resolution of the previously reported polyclonal gammopathy and gastrointestinal symptoms at the age of 6 years, repeat testing revealed normalization of nNO (Table ). Whole exome sequencing was subsequently obtained and detected a pathogenic mutation in the PIK3CD gene consistent with a diagnosis of immunodeficiency.

\section{Case 2}

A 4-year-old girl born at term and with no history of neonatal respiratory distress was evaluated for a year-round wet cough and rhinitis that started at 12 months of age. She had recurrent otitis media with myringotomy tubes placed around 3 years of age. She had normal oxygen saturation, scattered crackles and rhonchi on initial pulmonary exam and no digital clubbing. Previous work-up had included a normal sweat chloride test and two heterozygous VUS in DNAH5 on PCD genetic testing. Further genetic testing to confirm if the variants were in trans had not been completed. A sinus and chest CT had shown evidence of pansinusitis and mild bronchiectasis. Additional evaluation included low B and T cells and low immunoglobulin levels managed with intravenous immunoglobulin replacement therapy.

A repeat chest CT demonstrated progressive bronchial wall thickening and bronchiectasis, collapse of the right middle and upper lobes as well as extensive adenopathy. Bronchoscopy demonstrated lower airway inflammation, airway edema and increased mucus. Bronchoalveolar lavage showed neutrophil predominance on cell counts, however no organism was identified on culture. Initial $\mathrm{nNO}$ testing value of $38.9 \mathrm{~nL} / \mathrm{min}$ was in a range consistent with PCD. Considering the concurrent PCD and immunodeficiency concerns, repeat genetic testing confirmed prior VUS's in DNAH5 as well as a single pathogenic variant in PIK3CD. Her management included daily airway clearance, antibiotic prophylaxis and immunoglobulin replacement therapy.

\section{Discussion}

Both PCD and PID are part of the spectrum of suppurative lung diseases that describes individuals with persistent productive cough, recurrent pneumonia, and often bronchiectasis [3]. The PCD diagnostic testing guidelines acknowledge the importance of considering immunodeficiency testing especially when initial PCD genetic testing is not confirmatory[4]. Our cases further emphasize this recommendation, as both children presented with clinical symptoms that were consistent with PCD and with initial low nNO levels but were both later confirmed to have the same specific immunodeficiency.

The APDS1 is a primary immunodeficiency syndrome resulting from autosomal dominant heterozygous pathogenic variants. Affected individuals typically have abnormal lymphocyte subsets and develop recurrent sinopulmonary infections and often bronchiectasis. Other features include chronic diarrhea, recurrent viral infections and lymphadenopathy, as was noted in our second case, a clinical feature not routinely observed in PCD. Clinical features of PCD often overlap with those of PID. Organ laterality abnormalities and unexplained neonatal respiratory distress are more specific but not universal presentations of PCD and thus cannot always be used to differentiate from immunodeficiency. In individuals $>5$ years and with PCD, nNO values are persistently low [5] and thus the normalization in our first case was not consistent with a diagnosis of PCD. Confirmatory genetic testing and if needed ciliary EM studies following initial low nNO testing is recommended. When this testing fails to confirm a diagnosis of $\mathrm{PCD}$, the differential should be expanded to include immunodeficiency while nNO testing continues to be repeated.

These two cases are the first reports of APDS1 presenting as PCD with initial low nNO testing; however other immunodeficiencies presentations have been reported in a similar manner including GATA2, RAG1, and polysaccharide antibody deficiency $[2,6]$. These cases represent notable exceptions as there is evidence that nNO testing does have the ability to distinguish between PCD and many other cases of PID[6] and is still a useful diagnostic test, as illustrated in our first case in which the normalization of values prompted further work-up.

In conclusion, clinical features associated with PCD may overlap with PID and thus careful considerations 
are needed until a specific disease is confirmed. Nasal nitric oxide testing is a useful diagnostic tool in the work-up of individuals suspected to have PCD, but positive results need to be further confirmed with either genetic testing or ciliary EM. When PCD confirmatory testing is not conclusive, repeat nNO testing over time while considering an alternative diagnosis is strongly recommended.

\section{References}

1. Shapiro, A.J., et al., Diagnosis, monitoring, and treatment of primary ciliary dyskinesia: PCD foundation consensus recommendations based on state of the art review. Pediatr Pulmonol, 2016.51 (2): p. 115-32.

2. Barber, A.T., et al., Use caution interpreting nasal nitric oxide: Overlap in primary ciliary dyskinesia and primary immunodeficiency. Pediatr Pulmonol, 2021.

3. Redding, G.J. and E.R. Carter, Chronic Suppurative Lung Disease in Children: Definition and Spectrum of Disease. Front Pediatr, 2017.5 : p. 30.

4. Shapiro, A.J., et al., Limitations of Nasal Nitric Oxide Testing in Primary Ciliary Dyskinesia. Am J Respir Crit Care Med, 2020.202 (3): p. 476-477.

5. Shapiro, A.J., et al., Diagnosis of Primary Ciliary Dyskinesia. An Official American Thoracic Society Clinical Practice Guideline. Am J Respir Crit Care Med, 2018. 197 (12): p. e24-e39.

6. Zysman-Colman, Z.N., et al., Nasal Nitric Oxide in Primary Immunodeficiency and Primary Ciliary Dyskinesia: Helping to Distinguish Between Clinically Similar Diseases. J Clin Immunol, 2019.39 (2): p. 216-224.

\section{Hosted file}

Table 1 (1).docx available at https://authorea.com/users/405735/articles/557667-nasalnitric-oxide-may-not-differentiate-primary-ciliary-dyskinesia-from-certain-primaryimmunodeficiencies 